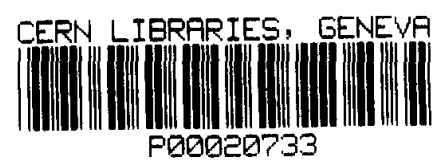

\title{
A class of C-integrable PDEs in multidimensions
}

\author{
F. Calogero \\ + Laboratoire de Physique Mathématique et Théorique, URA-CNRS 768 \\ Université de Montpellier II \\ 34095 Montpellier Cedex 5, France \\ ++ Dipartimento di Fisica, Università di Roma "La Sapienza" \\ Istituto Nazionale di Fisica Nucleare, Sezione di Roma, Italy
}

\section{Summary}

A simple algebraic mapping, when applied to linear PDEs, yields "C-integrable" nonlinear PDEs. Some examples are exhibited.

+ Visiting professor, November 1993

++ On leave while serving as Secretary General, Pugwash Conferences on Science and World Affairs, Geneva London Roma. 

1. Several years ago it was pointed out that the (nonlinear) mapping between the coefficients of a polynomial and its zeros provides a convenient technique to manufacture integrable dynamical systems [1]. The idea is to introduce a polynomial whose coefficients evolve linearly, and to then consider the evolution of its zeros, which is of course described by nonlinear equations. These evolution equations - some of which are rather neat [1] are therefore linearizable, and in some cases explicitly solvable, by "changing variables" (from the zeros of a polynomial to its coefficients). Hence, such models are instances of " $C$ integrable" equations, namely equations solvable via an appropriate Change of variables.

This approach [1], and a generalized version of it $[2,3]$, were used to generate several integrable dynamical systems; originally one-dimensional ones [1,3], and recently also multidimensional versions $[4,5]$.

In this paper we note that the same approach can also be used in the context of PDEs (rather than ODEs), and we thereby manufacture C-integrable nonlinear PDEs. Our treatment here is limited to exploiting the simple mapping between the zeros of a polynomial and its coefficients, namely an algebraic change of (dependent) variables; hence our results amount merely to an extension, from ODEs to PDEs, of the results of [1]. In fact, since our main purpose here is to present this idea rather than to exploit it exhaustively, we only exhibit below two representative examples; the interested reader will have no difficulty in manufacturing others, for instance by transcribing, after the manner outlined below, from ODEs to PDEs, other results of [1]. It is also possible to analogously transcribe, from the ODEs to PDE context, the more general results of [2-5]; but the interest of the nonlinear PDEs thus manufactured is moot.

The two examples of C-integrable PDEs explicitly treated in this paper read as follows:

$$
[\gamma \partial / \partial t-\Delta+V(\mathbf{r})] f_{j}(\mathbf{r}, t)=2 \sum_{k=1}^{n}{ }^{\prime}\left\{A^{2}+\left[\nabla f_{j}(\mathbf{r}, t)\right] \cdot\left[\nabla f_{k}(\mathbf{r}, t)\right]\right\} /\left[f_{j}(\mathbf{r}, t)-f_{k}(\mathbf{r}, t)\right], j=1, . ., n
$$

$$
\left[\square+M^{2}\right] f_{j}(\mathbf{r}, t)=2 \sum_{k=1}^{n} '\left\{A^{2}+f_{j, \mu}(\mathbf{r}, t) f_{k, \mu}(\mathbf{r}, t)\right\} /\left[f_{j}(\mathbf{r}, t)-f_{k}(\mathbf{r}, t)\right], \quad j=1, \ldots, n
$$

Notation: the $n$ scalar fields $f_{j}(\mathbf{r}, t)$ are the dependent variables ; the $\mathrm{N}$-dimensional "space" vector $\mathbf{r}$ and the "time" $t$ are the independent variables; $\nabla$ is the $\mathrm{N}$-dimensional gradient operator, $\Delta \equiv \nabla^{2}$ the Laplacian, $\square \equiv \partial^{2} / \partial t^{2}-\Delta$ the Dalembertian, $f_{j, \mu} \equiv$ $\partial f_{j} / \partial x_{\mu}, \mathbf{r} \equiv\left(x_{1}, . ., x_{N}\right), t \equiv i x_{0} ; V(\mathbf{r})$ is an arbitrary given function $; \gamma, A^{2}, M^{2}$ are arbitrary constants (which could be rescaled away) ; and the prime appended to the summation symbol signifies omission of the (singular) term with $k=j$. Of course in (2) the index $\mu$ is summed upon, from 0 to $N$; note that, for $N=3$, this is a relativisticallyinvariant equation in physical space-time. The number of fields $n$, and the dimensionality $N$ of space, are two arbitrary positive integers ; $n>1$ in order that the evolution equations be indeed nonlinear.

Clearly the quantity 


$$
F(\mathbf{r}, t)=\sum_{j=1}^{n} f_{j}(\mathbf{r}, t)
$$

satisfies the linear part of the equation, namely in the two cases there hold respectively

$$
\begin{gathered}
{[\partial / \partial t-\Delta+V(\mathbf{r})] F(\mathbf{r}, t)=0} \\
{\left[\square+M^{2}\right] F(\mathbf{r}, t)=0 .}
\end{gathered}
$$

Note that, for $N=3$, (5) is the standard Klein-Gordon equation, while for $\gamma=i(4)$ is the standard Schrödinger equation in the "external potential" $V(\mathbf{r})$, and for $\gamma=1$ and $V(\mathbf{r})=0(4)$ is the standard heat equation.

Below we indicate how the C-integrable equations (1) and (2) have been obtained, and how to solve the corresponding initial-value problems.

2. Firstly we report some useful formulas, relevant to both cases (and, as mentioned above, to many others as well).

Let $g(\mathbf{r}, t ; \lambda)$ be the polynomial of degree $n$ in $\lambda$, which has the quantities $f_{j}(\mathbf{r}, t), j=$ $1, . ., n$ as its $n$ zeros:

$$
g(\mathbf{r}, t ; \lambda)=\prod_{j=1}^{n}\left[\lambda-f_{j}(\mathbf{r}, t)\right] .
$$

It is then easily seen that there hold the following relations (subscripted variables denote partial differentiation):

$$
\begin{gathered}
\left(n g-\lambda g_{\lambda}\right) / g=\sum_{j=1}^{n}\left(\lambda-f_{j}\right)^{-1} f_{j}, \\
g_{\lambda \lambda} / g=2 \sum_{j=1}^{n}\left(\lambda-f_{j}\right)^{-1} \sum_{k=1}^{n}{ }^{\prime}\left(f_{j}-f_{k}\right)^{-1}, \\
g_{t} / g=-\sum_{j=1}^{n}\left(\lambda-f_{j}\right)^{-1} f_{j, t}, \\
g_{t t} / g=\sum_{j=1}^{n}\left(\lambda-f_{j}\right)^{-1}\left[-f_{j, t t}+2 \sum_{k=1}^{n}\left(f_{j}-f_{k}\right)^{-1} f_{j, t} f_{k, t}\right], \\
(\nabla g) / g=-\sum_{j=1}^{n}\left(\lambda-f_{j}\right)^{-1} \nabla f_{j}, \\
(\Delta g) / g=\sum_{j=1}^{n}\left(\lambda-f_{j}\right)^{-1}\left[-\Delta f_{j}+2 \sum_{k=1}^{n}\left(f_{j}-f_{k}\right)^{-1}\left(\nabla f_{j}\right) \cdot\left(\nabla f_{k}\right)\right] .
\end{gathered}
$$

We are now ready to treat the nonlinear evolution equations (1) and (2). 
3. We begin with the C-integrable equation (1). It is then easily seen, via (6) and (7), that $g(\mathbf{r}, t ; \lambda)$ satisfies the following linear PDE:

$$
\gamma g_{t}-\Delta g+V(\mathbf{r})\left[n g-\lambda g_{\lambda}\right]+A^{2} g_{\lambda \lambda}=0 .
$$

On the other hand set

$$
g(\mathbf{r}, t ; \lambda)=\sum_{m=0}^{n} \varphi_{m}(\mathbf{r}, t) \lambda^{n-m}
$$

Note that (6) and (9) imply

$$
\varphi_{0}(\mathbf{r}, t)=1
$$

and that they moreover yield a one-to-one correspondence between the $n$ "coefficients" $\varphi_{m}(\mathbf{r}, t), m=1, \ldots, n$, and the $n$ "zeros" $f_{j}(\mathbf{r}, t), j=1, \ldots, n$.

But (8) and (9) imply that the $n$ fields $\varphi_{m}(\mathbf{r}, t)$ evolve according to the following system of $n$ coupled linear PDEs:

$$
\gamma \varphi_{m, t}-\Delta \varphi_{m}+m V(\mathbf{r}) \varphi_{m}=\left(\delta_{1, m}-1\right)(n+2-m)(n+1-m) A^{2} \varphi_{m-2}, \quad m=1, \ldots, n .
$$

Note incidentally that, for $m=1$, the r.h.s. of this equation vanishes, consistently with (4) and with the relation $F(\mathbf{r}, t)=-\varphi_{1}(\mathbf{r}, t)$, which is clearly implied by (3), (6) and (9).

It is now clear that the initial value problem for (1) can be solved via the following 3 steps. (i) Given the initial data $f_{j}(\mathbf{r}, 0), j=1, \ldots, n$, compute $g(\mathbf{r}, 0 ; \lambda)$ from $(6)($ at $t=0)$, than $\varphi_{m}(\mathbf{r}, 0), m=1, \ldots, n$, from (9) (again at $t=0$ ). (ii) Then compute $\varphi_{m}(\mathbf{r}, t), m=$ $1, \ldots, n$, by solving recursively the system of linear PDEs (11), from $m=1$ upwards, using the initial data $\varphi_{m}(\mathbf{r}, 0)$ (for $m=2$, also use (10)). (iii) Finally, evaluate $g(\mathbf{r}, t ; \lambda)$ from (9) ; the zeros of this polynomial of degree $n$ in $\lambda$ yield then $f_{j}(\mathbf{r}, t)$, see (6).

We have omitted, for the sake of simplicity, to discuss, in step (ii), the role of boundary conditions. In this connection it is useful to note that the nonlinear mapping between the two sets $\left\{f_{j}, j=1, \ldots, n\right\}$ and $\left\{\varphi_{m} ; m=1, \ldots, n\right\}$ induced by (6) and (9) implies that to the set $\left\{f_{j}=0 ; j=1, \ldots, n\right\}$ there corresponds the set $\left\{\varphi_{m}=0 ; m=1, \ldots, n\right\}$; and, of course, viceversa.

4. The C-integrable equation (2) can be treated even more neatly. Indeed, via (6) and $(7)$, it clearly implies that $g(\mathbf{r}, t ; \lambda)$ satisfies the following linear PDE:

$$
\square g+M^{2}\left(n g-\lambda g_{\lambda}\right)+A^{2} g_{\lambda \lambda}=0 .
$$

It is now convenient to set

$$
g(\mathbf{r}, t ; \lambda)=\sum_{m=0}^{n} \varphi_{m}(\mathbf{r}, t) H_{n-m}(M \lambda / A),
$$

where $H_{m}(z)$ is the Hermite polynomial of degree $m$, so that

$$
H_{m}^{\prime \prime}(z)-z H_{m}^{\prime}(z)+m H_{m}(z)=0
$$




$$
\lim _{z \rightarrow \infty}\left[z^{-m} H_{m}(z)\right]=c_{m} .
$$

Here we leave open the choice of the normalization coefficients $c_{m}$.

Note that (6) and (13) imply (via (14b))

$$
\varphi_{0}(\mathbf{r}, t)=c_{n}^{-1}(A / M)^{n},
$$

and that they moreover yield a one-to-one correspondence between the $n$ fields $\varphi_{m}(\mathbf{r}, t), m=$ $1, \ldots, n$, and the $n$ fields $f_{j}(\mathbf{r}, t), j=1, \ldots, n$.

But (12) and (13) imply that the $n$ fields $\varphi_{m}(\mathrm{r}, t)$ evolve according to the following (decoupled!) linear PDEs:

$$
\square \varphi_{m}+m M^{2} \varphi_{m}=0, \quad m=1, \ldots, n .
$$

Note incidentally the consistency of this equation (with $m=1$ ) with (5), as implied by the relation $F(\mathbf{r}, t)=-c_{n-1}(M / A)^{n-1} \varphi_{1}(\mathbf{r}, t)$, which follows from (3), (6), (13) and (14b).

The initial value problem for $(2)$ is therefore solvable via the following 3 steps.

(i) Given the initial data, $f_{j}(\mathbf{r}, 0)$ and $f_{j, t}(\mathbf{r}, 0), j=1, \ldots, n$, compute $g(\mathbf{r}, 0 ; \lambda)$ (from (6) with $t=0$ ) and $g_{t}(\mathbf{r}, 0 ; \lambda)$ (from (7c) and (6), again with $t=0$ ). Then compute $\varphi_{m}(\mathbf{r}, 0)$ and $\varphi_{m, t}(\mathbf{r}, 0), m=1, \ldots, n$, from (13) and the time derivative of (13) (of course, both taken at $t=0$ ).

(ii) Integrate the Klein-Gordon equations (16), thereby computing $\varphi_{m}(\mathbf{r}, t)$ from the initial data $\varphi_{m}(r, 0)$ and $\varphi_{m, t}(r, 0)$. (Again, we ignore here the question of boundary conditions).

(iii) Compute $g(\mathbf{r}, t ; \lambda)$ from (13); its $n$ zeros then yield the $n$ fields $f_{j}(\mathbf{r}, t)$, see $(6)$.

5. Let us finally point out that the choice whether to introduce the coefficients $\varphi_{m}(\mathbf{r}, t)$ via (9) or (13) (or via some other analogous formula) is optional. Indeed, to deal with certain boundary conditions it might be expedient to use (9) rather than (13) also in the case of (2); or viceversa, for instance in the case of (1) with $V(\mathbf{r})=M^{2}$ independent of $\mathbf{r}$, it might be expedient to use (13) rather than (9), so has to decouple the system of linear PDEs satisfied by the coefficients $\varphi_{m}(\mathbf{r}, t)$. The derivation of the equations appropriate to treat such cases is left as a very easy exercise for the diligent reader. 


\section{REFERENCES}

[1] F. Calogero: Motion of poles and zeros of special solutions of nonlinear and linear partial differential equations, and related "solvable" many-body problems. Nuovo Cimento 43B, 177-241 (1978).

[2] F. Calogero: A class of solvable dynamical systems. Physica 18D, 280-302 (1986).

[3] F. Calogero: A class of integrable dynamical systems. Inverse Problems 1, L21-L24 (1985).

[4] F. Calogero: Interpolation in multidimensions, a convenient finite-dimensionel matrix representation of the (partial) differential operators, and some applications. J. Math. Phys. 34, 4704-4724 (1993).

[5] F. Calogero and Ji Xiaoda : Solvable (nonrelativistic, classical) n-body problems in multidimensions. I. J. Math. Phys. (in press). 
